\title{
Desarrollo didáctico complementario de las expresiones analíticas de las acciones de sacudimiento en las máquinas de combustión interna
}

\section{Complementary didactic development of analytical expressions of shaking actions in internal combustion machines}

\author{
VÁZQUEZ-GONZÁLEZ, Benjamín†๋*, JIMÉNEZ-RABIELA, Homero, RAMÍREZ-CRUZ, José Luis \\ y GARCÍA-SEGURA, Pedro
}

Universidad Autónoma Metropolitana, Unidad Azcapotzalco, División de Ciencias Básicas e Ingeniería, Departamento de Energía, Av. San Pablo 180 Colonia Reynosa Tamaulipas, Azcapotzalco, Ciudad de México, C. P. 02200

ID $1^{\text {er }}$ Autor: Benjamín, Vázquez-González / ORC ID: 0000-0002-9030-5662, Researcher ID Thomson: S-2417-2018, CVU CONACYT ID: 25749

ID $1^{\text {er }}$ Coautor: Homero, Jiménez Rabiela / Researcher ID Thomson: S-2299-2018, CVU CONACYT ID: 123386

ID $2^{\text {do }}$ Coautor: José Luis, Ramírez Cruz / ORC ID: 0000-0003-0762-2630, Researcher ID Thomson: G-3405-2019, CVU CONACYT ID: 921268

ID $3^{\text {er }}$ Coautor: Pedro, García Segura / ORC ID: 0000-0003-4947-084X, Researcher ID Thomson: S-2360-2018, CVU CONACYT ID: 371233

DOI: $10.35429 / J T A E .2019 .8 .3 .16 .26$

Recibido: 09 de Abril, 2019; Aceptado 30 de Junio, 2019

\section{Resumen}

El estudio de las fuerzas y los pares de sacudimiento que se presentan en mecanismos, son de principal interés en el estudio de la dinámica de las máquinas de combustión interna. Una máquina de combustión interna se modela a partir de una disposición particular de un conjunto de mecanismos del tipo biela-manivela-corredera. Durante el desempeño de la máquina de combustión interna, el movimiento repetitivo de la corredera o pistón induce aceleraciones y fuerzas que se transmiten y distribuyen entre la biela y la manivela o cigüeñal. Cuando el cigüeñal se ve sometido a las componentes de las fuerzas inducidas por el pistón, se generan a su vez los pares de sacudimiento. El análisis de los pares de sacudimiento tiene como fin, determinar los valores dinámicos de los contrapesos que se deben adicionar a cada mecanismo, con el objetivo de reducir el conjunto de las acciones de sacudimiento. En la bibliografía tradicional en la que se presentan las ecuaciones dinámicas no siempre se presentan los desarrollos analíticos completos, existen referencias que presentan resultados parciales, en este trabajo se presentan los desarrollos algebraicos que permiten validar la correspondencia entre las expresiones de distintas fuentes bibliográficas en la enseñanza de estos temas.

Didáctica, Acciones de sacudimiento, Máquinas de combustión interna

\begin{abstract}
The study of the forces and the pairs of shaking that appear in mechanisms, are of main interest in the study of the dynamics of the internal combustion engines. An internal combustion engine is modeled from a particular arrangement of a set of mechanisms of the connecting-rodcrank type. During the performance of the internal combustion engine, the repetitive movement of the slide or piston induces accelerations and forces that are transmitted and distributed between the connecting rod and the crank or crankshaft. When the crankshaft is subjected to the components of the forces induced by the piston, the shaking torques are generated in turn. The purpose of the analysis of the pairs of shaking is to determine the dynamic values of the counterweights that must be added to each mechanism, with the aim of reducing the set of shaking actions. In the traditional bibliography in which the dynamic equations are presented, the complete analytical developments are not always presented; there are references that present partial results, in this work the algebraic developments are presented that allow to validate the correspondence between the expressions of different bibliographic sources in the teaching of these subjects.
\end{abstract}

Didactics, Shaking actions, Internal combustion engine

Citación: VÁZQUEZ-GONZÁLEZ, Benjamín, JIMÉNEZ-RABIELA, Homero, RAMÍREZ-CRUZ, José Luis y GARCÍASEGURA, Pedro. Desarrollo didáctico complementario de las expresiones analíticas de las acciones de sacudimiento en las máquinas de combustión interna. Revista de Tecnología y Educación. 2019. 3-8: 16-26

\footnotetext{
* Correspondencia del Autor (Correo electrónico: bvg@ correo.azc.ua.mx)

$\dagger$ Investigador contribuyendo como primer autor.
} 


\section{Introducción}

El cambio climático ha producido que se considere sensiblemente la sustitución de vehículos a base de combustión, por vehículos alternos. En su artículo, Menchaca (2013), menciona que en el Área Metropolitana de Monterrey, los vehículos de combustión fueron responsables de la emisión a la atmósfera del 99\% del monóxido de carbono, y esto motiva el desarrollo de nuevas tecnologías, tales como el desarrollo de vehículos híbridos. Sin embargo, el transporte de mercancías a gran escala se realiza por medio del transporte marítimo o por tren, y en este contexto el desarrollo tecnológico tiene una perspectiva distinta.

Existen avances importantes, por ejemplo, ABB (2016) informa sobre el desarrollo de un sistema de propulsión eléctrica de buques. Este sistema coadyuva en mejorar el desempeño de los motores a base de combustible, pero no los sustituye. Otras alternativas consideran producir electricidad dentro del buque con motores de combustión interna, para después alimentar motores eléctricos, que se conectan directamente a las hélices de los buques.

Estos estudios permiten asegurar que sigue estando vigente el estudio de las máquinas de combustión interna, así como la búsqueda de mejoras en su desempeño.

En este trabajo se presentan y discuten los desarrollos que la bibliografía tradicional ofrece sobre temas relacionados con los fundamentos de la dinámica de máquinas.

Parte importante del estudio de las máquinas de combustión interna, radica en el análisis y la determinación de las fuerzas y los pares de sacudimiento, donde el objetivo posterior es el estudio para disminuir estas acciones. De manera tradicional este estudio se inicia, determinando las fuerzas internas que se producen durante el desempeño de un mecanismo biela-manivela-corredera, y posteriormente el par de sacudimiento ocasionado por las mismas fuerzas. Esto viene acompañado de un modelo cinemático del mecanismo, en el que se considera que los elementos conductores del movimiento, los eslabones, son simples cuerpos rígidos ideales.
Posteriormente se determinan modelos dinámicos en los que participa la distribución de la masa y finalmente se determinan las fuerzas de sacudimiento, para un modelo dinámico del eslabonamiento o mecanismo. Norton (2009) presenta desarrollos relativamente compactos, a su vez incluye a las fuerzas inerciales y las relaciona con las fuerzas de explosión de los gases de combustión, finalmente obtiene las expresiones representativas a las fuerzas de sacudimiento.

Wilson (2002), presentan un desarrollo alterno, realizando un análisis directo entre las fuerzas internas, obteniendo finalmente las acciones de sacudimiento, otros autores siguen alguno de estos desarrollos, Cleghorn (2005), Erdman, et. al. (2001). En este trabajo se desarrollan las expresiones desde una perspectiva más directa y completa, aportando un manejo extendido de los desarrollos algebraicos, siguiendo la metodología de Wilson (2002), y se llega a las expresiones de Norton (2009).

El objetivo de este trabajo es presentar un desarrollo completo para la obtención de las fuerzas de sacudimiento, basado en el análisis dinámico utilizando las Leyes de Newton. La metodología consiste en desarrollar los diagramas de cuerpo libre de cada componente del mecanismo biela-manivela-corredera, ilustrado detalladamente con gráficas las acciones dinámicas presentes.

En la enseñanza de estos temas, es importante y necesario proporcionar un desarrollo continuo, que ofrezca una descripción ininterrumpida del acoplamiento de las ecuaciones, así como de las aproximaciones que se obtienen y la validez de estas. Con lo anterior se contribuye en el contexto fundamental de la educación, pero también en el manejo de expresiones analíticas. En este trabajo se determinan las expresiones del sacudimiento por dos metodologías, una de ellas reportada en la literatura, la otra por desarrollos y descripciones propias.

\section{Modelo cinemático}

Esta sección se inicia con el análisis cinemático de un mecanismo biela-manivela-corredera, para posteriormente obtener un modelo dinámico. 
A continuación se muestra la figura que ilustra la configuración cinemática de un mecanismo biela-manivela-corredera.

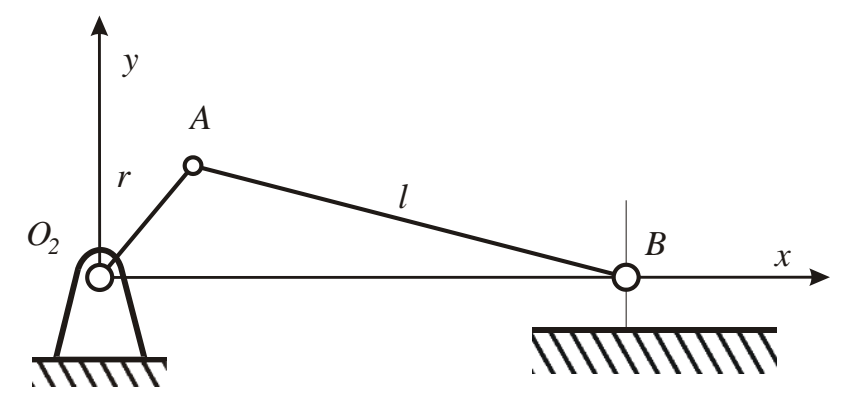

Figura 1 Representación cinemática de un mecanismo biela-manivela-corredera.

La manivela corresponde con el eslabón denominado $r$ que viene a ser parte del cigüeñal, al integrar un motor de combustión interna. La biela se denota con la variable $l$, y la corredera o pistón con el punto $B$. Por ser un modelo cinemático, no se consideran en este momento las masas de los cuerpos mencionados.

Este mecanismo al ser parte de una máquina de combustión interna, tiene su movimiento originado en el punto $B$, el cual se desplaza por la acción de la explosión, resultado de la combustión y esto produce la rotación la manivela $r$. Para determinar las relaciones de posición mencionadas anteriormente, considérese la siguiente figura.

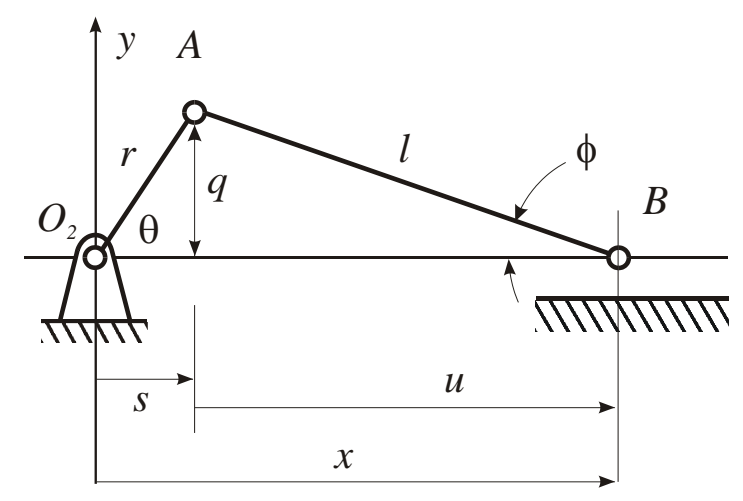

Figura 2 Parámetros de posición del mecanismo bielamanivela-corredera

De la Figura 2, se pueden construir las siguientes relaciones geométricas.

$q=r \operatorname{sen} \theta=l \operatorname{sen} \phi$

donde

$\theta=\omega t$
Esta expresión permite conocer la posición de la manivela, para un instante determinado $t$, dada una velocidad angular $\omega$.

igualdades,

También se distinguen las siguientes

$s=r \cos \theta$

$u=l \cos \phi$

$x=s+u=r \cos \omega t+l \cos \phi$

De la ecuación (1) y (2) resulta,

$\operatorname{sen} \phi=\frac{r}{l} \operatorname{sen} \omega t$

De la identidad trigonométrica

$(\operatorname{sen} \phi)^{2}+(\cos \phi)^{2}=1$

se obtiene,

$$
\begin{aligned}
\cos \phi & =\sqrt{1-(\operatorname{sen} \phi)^{2}} \\
& =\sqrt{1-\left(\frac{r}{l} \operatorname{sen} \omega t\right)^{2}}
\end{aligned}
$$

Por tanto, la posición $x$ del punto $B$ con respecto al punto fijo $\mathrm{O}_{2}$ es,

$x=r \cos \omega t+l \sqrt{1-\left(\frac{r}{l} \operatorname{sen} \omega t\right)^{2}}$

La ecuación (7) proporciona una relación entre la velocidad de rotación de la manivela y la posición instantánea del pistón.

Otra expresión importante es la que permite conocer las relaciones de posición angular entre la manivela y la biela, esta se establece a partir de la expresión,

$\tan \phi=\frac{\operatorname{sen} \phi}{\cos \phi}=\frac{{ }_{l}^{r} \operatorname{sen} \omega t}{\sqrt{1-\left(\frac{r}{l} \operatorname{sen} \omega t\right)^{2}}}$

En el contexto de la cinemática, ahora es posible determinar una relación de la aceleración del punto $B$, desde el punto fijo $O_{2}$.

La derivada con respecto al tiempo de la ecuación (7), resulta,

$\dot{x}=-r \omega\left(\operatorname{sen} \omega t+\frac{r(\operatorname{sen} 2 \omega t)}{2 l \sqrt{1-\left(\frac{r}{l} \operatorname{sen} \omega t\right)^{2}}}\right)$ 
La aceleración del pistón localizado en el punto $B$, se determina al derivar la ecuación (9), se reporta el resultado final,

$$
\begin{aligned}
\ddot{x}= & -r \omega^{2}(\cos \omega t- \\
& \left.\frac{r\left(l^{2}\left(1-2 \cos ^{2} \omega t\right)\right)-r^{2} \operatorname{sen}^{4} \omega t}{\left(l^{2}-(r \operatorname{sen} \omega t)^{2}\right)^{\frac{3}{2}}}\right)
\end{aligned}
$$

Algunos autores como Uicker et. al, (2003) realizar una primera aproximación en el radical del primer término de la ecuación (7), lo que lleva a un cálculo más rápido, para no restar generalidad en este momento, se presenta la ecuación (10). Se ha mostrado lo complejas que son las expresiones de la velocidad y la aceleración y estas pueden ser útiles para análisis más completos posteriormente, sin embargo, siguiendo las pautas de Norton (2009) $\mathrm{y}$ otros autores, ahora se toma el siguiente paso, obtener una aproximación de la posición $x$ y evaluar tanto la velocidad como la aceleración. Se utiliza la aproximación propuesta por la serie del binomio, esto es, recordando la serie binomial siguiente,

$$
\begin{aligned}
& (a+b)^{n}=a^{n}+n a^{n-1} b+\frac{n(n-1)}{2 !} a^{n-2} b^{2}+ \\
& \frac{n(n-1)(n-2)}{3 !} a^{n-3} b^{3}
\end{aligned}
$$

El radical de la ecuación (7), correspondiente al desplazamiento, se puede escribir,

$$
\sqrt{1-\left(\frac{r}{l} \operatorname{sen} \omega t\right)^{2}}=\left(1-\left(\frac{r}{l} \operatorname{sen} \omega t\right)^{2}\right)^{\frac{1}{2}}
$$

Desarrollando conforme la aproximación binomial, para los primeros tres términos, se tiene,

$$
\begin{aligned}
& \left(1-\left(\frac{r}{l} \operatorname{sen} \omega t\right)^{2}\right)^{\frac{1}{2}} \cong 1^{\frac{1}{2}}+ \\
& \left(\frac{1}{2}\right) 1^{\frac{1}{2}-1}\left(-\left(\frac{r}{l} \operatorname{sen} \omega t\right)^{2}\right)+ \\
& \frac{\frac{1}{2}\left(\frac{1}{2}-1\right)}{2 !} 1^{\frac{1}{2}-2}\left(-\left(\frac{r}{l} \operatorname{sen} \omega t\right)^{2}\right)^{2}
\end{aligned}
$$

lo cual se simplifica a

$$
\begin{aligned}
& \left(1-\left(\frac{r}{l} \operatorname{sen} \omega t\right)^{2}\right)^{\frac{1}{2}} \cong 1-\frac{1}{2}\left(\frac{r}{l} \operatorname{sen} \omega t\right)^{2}- \\
& \frac{1}{8}\left(\frac{r}{l} \operatorname{sen} \omega t\right)^{4}
\end{aligned}
$$

Esta expresión será considerada como la expresión de la posición $x$ del pistón con respecto al punto $\mathrm{O}_{2}$. El desarrollo anterior permite observar lo pequeñas que son las contribuciones del tercer término, por lo que se emplean sólo los dos primeros, esto es,

$x \cong r \cos \omega t+l\left(1-\frac{1}{2}\left(\frac{r}{l} \operatorname{sen} \omega t\right)^{2}\right)$

Ahora es conveniente desarrollar un modelo dinámico del mecanismo bielamanivela-corredera. Esto implica considerar los atributos de la masa de cada eslabón del mecanismo. El análisis convencional de las fuerzas de sacudimiento, se inicia en base a un modelo, en el que las masas se localizan en el centro de masa de los eslabones, posteriormente se desarrolla un modelo aproximado de masas distribuidas en los extremos de los eslabones y finalmente se realiza nuevamente un análisis dinámico con el modelo dinámico de masas distribuidas. La propuesta de este trabajo, considera iniciar el análisis de sacudimiento, partiendo del análisis de un modelo de masas distribuidas en los extremos de los eslabones y posteriormente, realizar el análisis de fuerzas de sacudimiento.

Para ilustrar lo mencionado con anterioridad, considérese la siguiente figura.

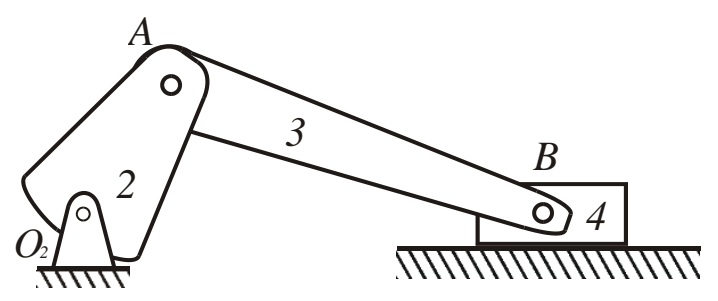

Figura 3 Configuración dinámica del mecanismo bielamanivela-corredera

En la Figura 1 se ilustró un mecanismo biela-manivela-corredera en el que los eslabones se componen de barras rígidas sin masa, el estudio de la fuerzas de sacudimiento requiere conocer la distribución de las masas. En la Figura 3, se ha incluido ésta propiedad, mostrando eslabones con forma determinada, lo que implica considerar los respectivos momentos de inercia.

VÁZQUEZ-GONZÁLEZ, Benjamín, JIMÉNEZ-RABIELA, Homero, RAMÍREZ-CRUZ, José Luis y GARCÍA-SEGURA, Pedro. Desarrollo didáctico complementario de las expresiones analíticas de las acciones de sacudimiento en las máquinas de combustión interna. Revista de Tecnología y Educación. 2019 


\section{Modelo dinámico aproximado del mecanismo biela-manivela-corredera}

En esta sección se estudia directamente el modelo dinámico del mecanismo, esto incluye determinar un modelo analítico, partiendo de la distribución de la masa de los eslabones.

En la Figura 4, se muestra un esquema de la biela, en la Figura 4 a), se representa la biela como un cuerpo rígido homogéneo, su centro de masa localizado a la distancia $l_{a}$ del punto $A$. En las Figuras 4 b) a d) se representan las etapas para la determinación del modelo aproximado de dos masas en los extremos. El esquema de la Figura 4, propuesto por Norton (2009), es muy útil desde el punto de vista didáctico, porque permite visualizar la diferencia entre el modelo dinámico exacto y el aproximado.

A continuación se presenta el desarrollo analítico.

El modelo genérico considera que la masa total de la biela se puede concentrar en dos puntos y que se conserva la magnitud de su momento de inercia.

a) Biela original
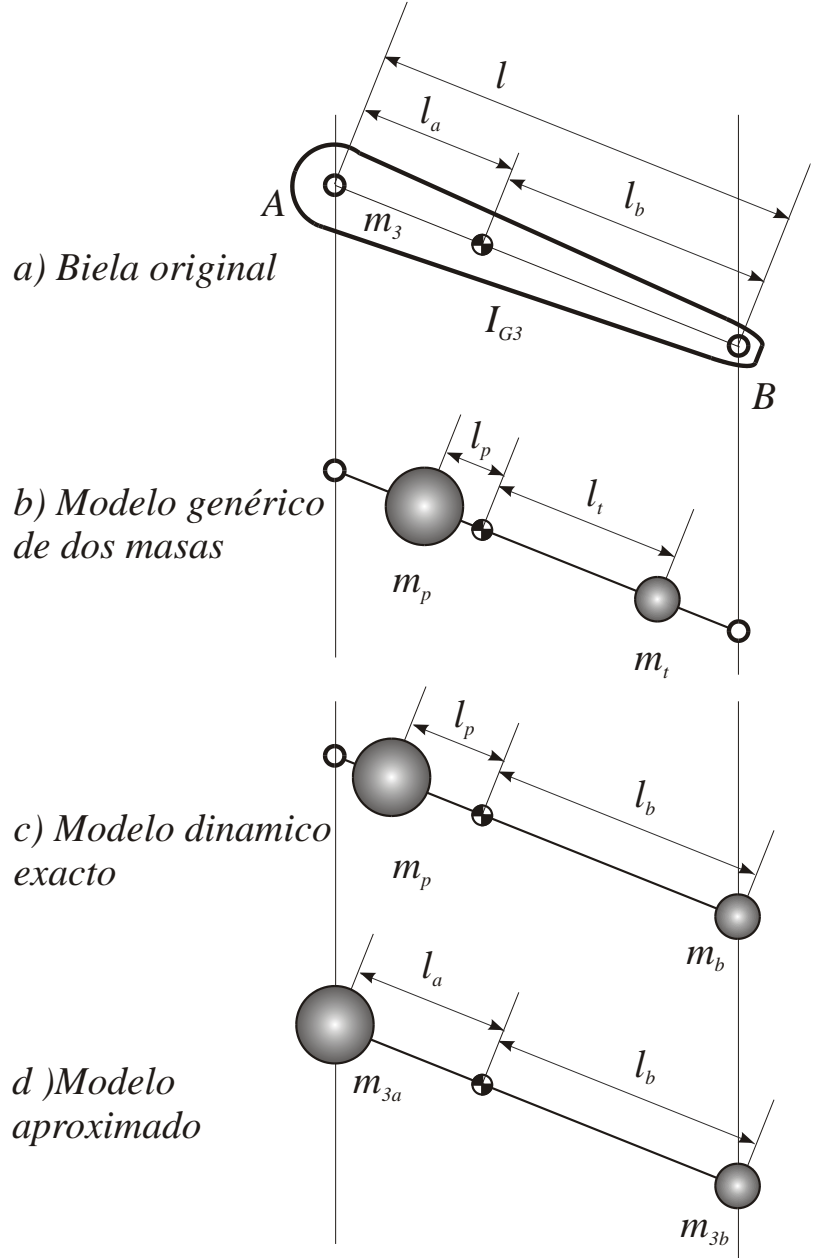

Figura 4 Esquema representativo del modelo dinámico de la biela para dos masas puntuales, colocadas en los extremos
Para determinar el modelo genérico se debe conservar la masa, esto es,

$m_{3}=m_{p}+m_{t}$

donde la ecuación (16) expresa que la masa total se distribuirá en dos masas puntuales $m_{p}$ y $m_{t}$ a determinar.

También se debe conservar la posición del centro de masa, esto es,

$M \bar{r}=\sum_{1}^{2} m_{i} r_{i}$

Donde la condición para que el centro de masa conserve su posición se representa por $\bar{r}=0$, esto es, la posición del centro de masa, medida desde el centro de masa mismo.

Por tanto,

$M \bar{r}=-m_{p} l_{p}+m_{t} l_{t}=0$

de donde resulta

$m_{p} l_{p}=m_{t} l_{t}$

$l_{t}$ es una variable que se elige arbitrariamente para balancear el centro de masa, con esto es posible fijar a $m_{p}$ en la posición $l_{p}$.

Cómo se observa esto da libertad de elegir una distancia donde colocar a una de las dos masas, en las que se distribuye la masa total de la biela, véase la Figura 4 b).

Con esta libertad se realiza la siguiente designación, $b=t$, esto es, se colocará a la masa $m_{t}$ en el punto $b$, por tanto $m_{b}=m_{t}$, véase la Figura 4 c).

Así que la ecuación (16) se convierte en

$m_{3}=m_{p}+m_{b}$

Lo anterior permite determinar la posición de $m_{p}$, para una masa determinada $m_{3}$.

Cómo,

$m_{p} l_{p}=m_{b} l_{b}$ 
$m_{p}=\frac{m_{b} l_{b}}{l_{p}}$

Ahora,

$$
\begin{aligned}
m_{3} & =\frac{m_{b} l_{b}}{l_{p}}+m_{b}=\frac{m_{b} l_{b}}{l_{p}}+\frac{l_{p}}{l_{p}} m_{b} \\
& =\left(\frac{l_{b}}{l_{p}}+\frac{l_{p}}{l_{p}}\right) m_{b}=\left(\frac{l_{b}+l_{p}}{l_{p}}\right) m_{b}
\end{aligned}
$$

o bien,

$$
m_{b}=\frac{l_{p}}{l_{b}+l_{p}} m_{3}
$$

De la ecuación (22) se obtiene,

$$
m_{p}=\frac{m_{b} l_{b}}{l_{p}}=\left(\frac{l_{p}}{l_{b}+l_{p}} m_{3}\right) \frac{l_{b}}{l_{p}}=\frac{l_{b}}{l_{b}+l_{p}} m_{3}
$$

La última condición dinámica para obtener un modelo de dos masas equivalente para un cuerpo rígido, es la conservación del momento de inercia, esto es,

$$
I_{G 3}=m_{p} l_{p}^{2}+m_{t} l_{t}^{2}=m_{p} l_{p}^{2}+m_{b} l_{b}^{2}
$$

Sustituyendo lo valores de $m_{p}$ y $m_{b}$ de las ecuaciones (24) y (25), resulta

$$
I_{G 3}=m_{3} l_{p} l_{b}
$$

Finalmente, es posible determinar la posición $l_{p}$ para un cuerpo específico, si se conoce su momento de inercia, esto es,

$l_{p}=\frac{I_{G 3}}{m_{3} l_{b}}$

Conforme a la Figura 4 c), se tiene un modelo exacto de dos masas puntuales. El modelo aproximado se obtiene cuando se define de manera arbitraria lo siguiente, $l_{p}=l_{a}$.

Por tanto, el modelo aproximado establece los valores de las masas en los extremos y las distancias respectivas, esto es,

$m_{3 a}=m_{3} \frac{l_{b}}{l_{b}+l_{a}}, m_{3 b}=m_{3} \frac{l_{a}}{l_{b}+l_{a}}$

En esta sección se han desarrollado los procedimientos planteados en la bibliografía, que por lo general no se muestran y aquí se realiza un desarrollo completo.
Para determinar el modelo aproximado de la manivela se realiza un procedimiento semejante.

En la Figura 5 se muestra un esquema de cuerpo rígido de la manivela. En la Figura 5 a) se tiene el cuerpo rígido original y en la Figura 5 b), el modelo aproximado de dos masas. Se sigue el procedimiento anterior.

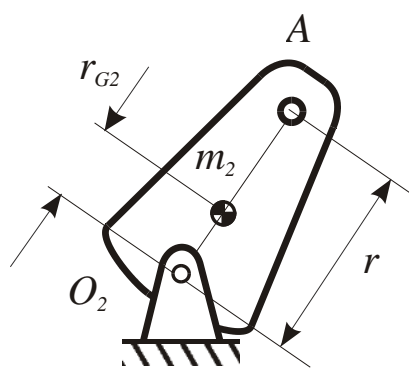

(a)

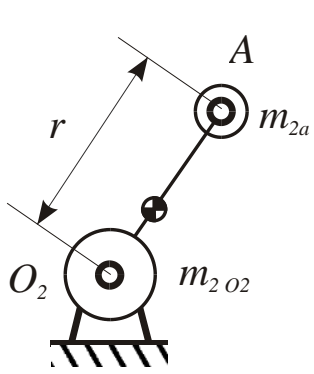

(b)
Figura 5 Esquema representativo del modelo dinámico de la manivela para dos masas puntuales, colocadas en los extremos

Sin embargo, en este caso por el tipo de movimiento de rotación pura con velocidad angular constante del cuerpo con respecto al punto $\mathrm{O}_{2}$, lleva a que la condición del momento de inercia no se considere.

En esta situación, se tendrán dos masas puntuales, una localizada en el punto $\mathrm{O}_{2}$ y otra en el punto $A$, esto es,

$m_{2 a}=m_{2} \frac{r_{G 2}}{r}$

La masa localizada en $\mathrm{O}_{2}$,

$m_{2 O 2}=m_{2 a} \frac{r_{2}}{r_{G 2}}$

es una masa puntual rotando con velocidad constante en torno a $\mathrm{O}_{2}$.

En la siguiente figura se presenta el modelo final del mecanismo biela-manivelacorredera de masas puntuales. 


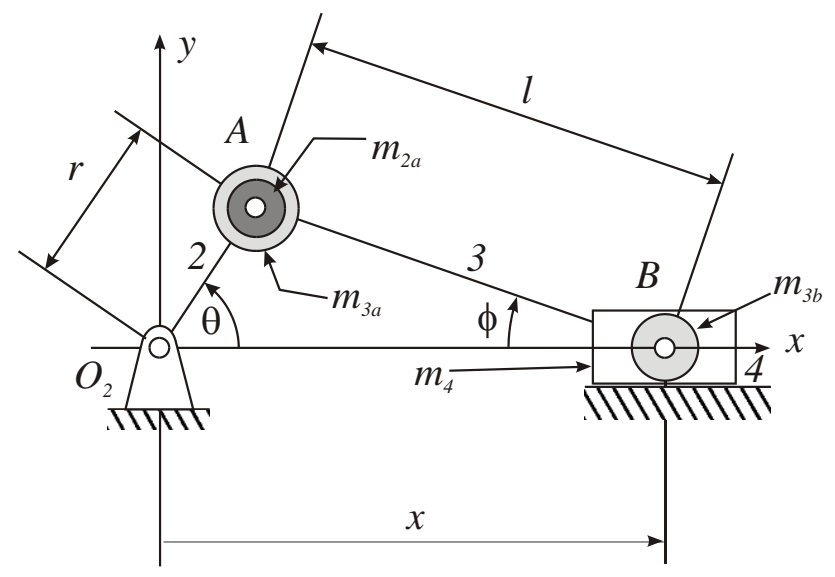

Figura 6 Esquema representativo del modelo dinámico del mecanismo biela-manivela-corredera de masas puntuales

En la Figura 6, se ilustra el modelo final del mecanismo biela-manivela-corredera con masa puntuales colocadas en los extremos, como se ha mencionado es un modelo de masas puntuales aproximado. Se observa que se cumplen las condiciones inerciales en los puntos $A$ y $B$, respectivamente,

$m_{A}=m_{2 a}+m_{3 a}, m_{B}=m_{3 b}+m_{4}$

\section{Fuerzas internas del mecanismo}

Para determinar las acciones que actúan sobre el mecanismo ahora se realiza un análisis dinámico de fuerzas, se debe realizar el diagrama de cuerpo libre de cada eslabón del mecanismo.

\section{Manivela}

En la siguiente figura se muestra el diagrama de cuerpo libre del eslabón 2, modelado como un conjunto de masas puntuales, la figura ilustra las componentes de la fuerza $F_{32}$ que producen par dinámico, estas componentes son perpendiculares al vector $r$ de la masa $m_{A}$.

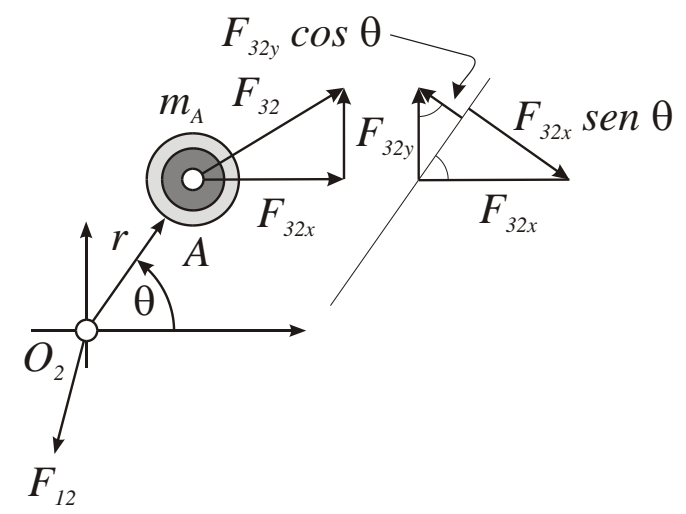

Figura 7 Modelo de dos masas puntuales de la manivela
La masa $m_{A}$ se encuentra en rotación pura, con velocidad angular es constante.

El balance de fuerzas para este eslabón es cero, puesto que la masa no se desplaza, sin embargo, las reacciones a que está sujeta, producen pares de rotación con respecto al punto $\mathrm{O}_{2}$, esto es,

$\sum M_{O 2}=-F_{32 x} r \operatorname{sen} \theta+F_{32 y} r \cos \theta+T_{21}=I_{2} \alpha_{2}$

resultando

$T_{12}=F_{32 x} r \operatorname{sen} \theta-F_{32 y} r \cos \theta$

donde $\alpha_{2}=0$ y $T_{12}=-T_{21}$.

Nótense los signos opuestos en la evaluación de los pares de rotación, producidos por las componentes de fuerza de reacción $F_{32}$, que corresponde con la fuerza que el eslabón 3 produce sobre el 2.

Esta ecuación ha permitido obtener de manera directa el par de sacudimiento $T_{12}$.

\section{Biela}

Ahora se evalúan las acciones que percibe el eslabón 3 o la biela. En la siguiente figura se muestra su diagrama de cuerpo libre.

La Figura 8 muestra una esmerada descripción para identificar las componentes que producen pares de rotación.

Para identificar con facilidad estas fuerzas, primero obsérvese que el ángulo $\phi$ lo desarrolla la biela con respecto a la horizontal.

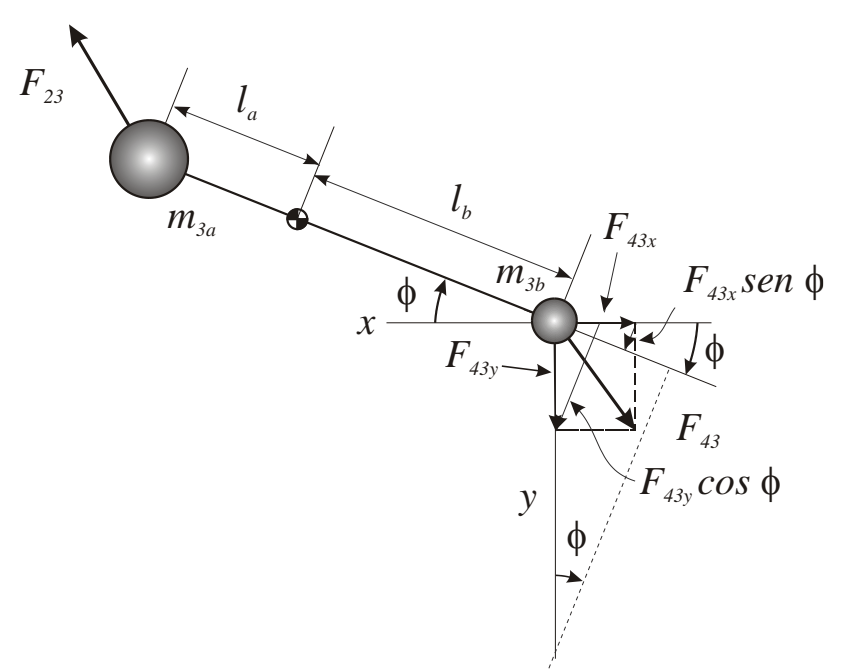

Figura 8 Modelo de dos masas puntuales de la biela

VÁZQUEZ-GONZÁLEZ, Benjamín, JIMÉNEZ-RABIELA, Homero, RAMÍREZ-CRUZ, José Luis y GARCÍA-SEGURA, Pedro. Desarrollo didáctico complementario de las expresiones analíticas de las acciones de sacudimiento en las máquinas de combustión interna. Revista de Tecnología y Educación. 2019 
Se puede ver que al lado derecho de la masa $m_{3 b}$, dicho ángulo ahora se presenta entre la horizontal y una línea auxiliar que tiene la misma dirección que la línea de la biela. Por triángulos semejantes también se observa que el ángulo $\phi$ se forma entre la vertical y una línea auxiliar perpendicular a la dirección de la línea de la biela.

La fuerza $F_{43}$, es la fuerza de reacción que produce el pistón o eslabón 4 sobre la biela (eslabón 3), las componentes de ésta fuerza sobre los ejes $x-y$, producirán pares de rotación. Estas componentes son perpendiculares a la dirección de la línea de la biela, por tanto los pares de rotación de la fuerza $F_{43}$.

Por otro lado, la biela modelada por un conjunto de dos masas, desarrolla aceleración, por la acción de la fuerzas y también por describir un movimiento en el plano. Entonces la ecuación del par de rotación de la biela, con respecto al punto $A$, se puede expresar por,

$$
\begin{aligned}
\sum M_{A} & =F_{43 x} l \operatorname{sen} \phi+F_{43 y} l \cos \phi \\
& =m_{3 b} a_{B x} l \operatorname{sen} \phi
\end{aligned}
$$

Nótese que se está evaluando los pares con respecto al punto $A$, por tanto la masa $m_{A}$, no es parte de esta expresión.

De esta ecuación se despeja $F_{43 y}$, el resultado es

$F_{43 y}=\left(m_{3 b} a_{B x}-F_{43 x}\right) \tan \phi$

La biela también desarrolla fuerzas, porque este cuerpo se encuentra desplazándose en el plano $x-y$.

Las fuerzas en las direcciones $x$ y $y$ respectivamente son,

$\sum F_{3 x}=F_{23 x}+F_{43 x}=m_{A} a_{A x}+m_{3 b} a_{B x}(37)$

$\sum F_{3 y}=F_{23 y}+F_{43 y}=m_{A} a_{A y}$

Este balance de fuerzas se obtiene a partir del diagrama de cuerpo libre de la Figura 8.

\section{Pistón}

Finalmente en la siguiente figura se desarrolla el diagrama de cuerpo libre para el eslabón 4 o pistón.
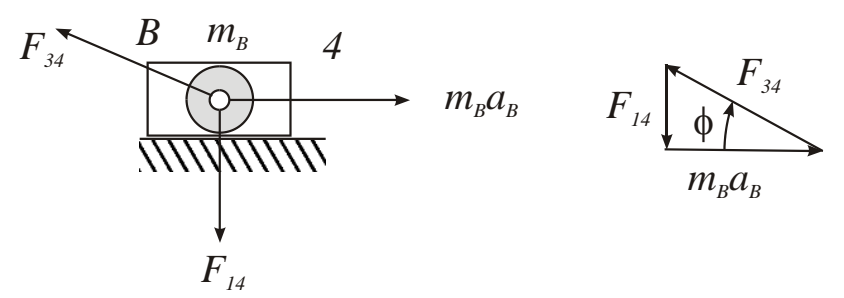

Figura 9 Diagrama de cuerpo libre del eslabón 4 o pistón

Por ser una masa puntual su análisis es muy sencillo, el pistón no desarrolla movimiento vertical, porque su función es sólo de deslizamiento horizontal. Entonces el balance de fuerzas resulta ser,

$\sum F_{4 x}=F_{34 x}=m_{4} a_{B x}$

Las fuerzas de reacción cumplen con la Tercera Ley de Newton, por tanto, desde la perspectiva del eslabón 3 , se tiene que,

$F_{34 x}=-F_{43 x}$

Finalmente, resulta,

$-F_{43 x}=m_{4} a_{B x}$

\section{Fuerzas inerciales}

Para dar un contexto de la acción de las fuerzas desde la perspectiva de Newton, definimos la expresión,

$F_{e}=m a$

que expresa el movimiento de las masas por las fuerzas externas.

La ecuación (42) se puede escribir como,

$F_{e}-m a=0$

y definiendo,

$F_{i}=-m a$

resulta,

$F_{i}+F_{e}=0$

VÁZQUEZ-GONZÁLEZ，Benjamín, JIMÉNEZ-RABIELA，Homero, RAMÍREZ-CRUZ, José Luis y GARCÍA-SEGURA, Pedro. Desarrollo didáctico complementario de las expresiones analíticas de las acciones de sacudimiento en las máquinas de combustión interna. Revista de Tecnología y Educación. 2019 
con esto, se expresa que las fuerzas externas $F_{e}$ son opuestas a las fuerzas inerciales $F_{i}$.

En el contexto de las máquinas de combustión interna, las fuerzas externas son las producidas por los gases de combustión y las internas, las que se desarrollan entre los eslabones del mecanismo.

\section{Fuerzas de sacudimiento}

Las fuerzas de sacudimiento se definen como aquellas que se presentan directamente en el eslabón fijo, por acción de los elementos móviles, Norton (2009).

La fuerza que se presenta en el eslabón fijo es una fuerza inercial, por tanto, se puede establecer que

$$
\begin{aligned}
& -F_{i x}=F_{e x}=F_{23 x} \\
& -F_{i y}=F_{e y}=F_{23 y}+F_{43 y}
\end{aligned}
$$

Ahora se desarrollan las expresiones de las fuerzas inerciales, $F_{32}, F_{32 y}$ y $F_{34 y}$. La componente $F_{23 x}$ se puede expresar en términos de las ecuaciones (37) y (39),

$$
\begin{aligned}
F_{23 x} & =m_{A} a_{A x}+m_{3 b} a_{B x}+m_{4} a_{B x} \\
& =m_{A} a_{A x}+\left(m_{3 b}+m_{4}\right) a_{B x}
\end{aligned}
$$

Sustituyendo la ecuación (36) en la (38), se obtiene, la componente $F_{23 y}$, esto es,

$$
\begin{aligned}
F_{23 y} & =m_{A} a_{A y}-F_{43 y} \\
& =m_{A} a_{A y}-\left(m_{3 b} a_{B x}-F_{43 x}\right) \tan \phi
\end{aligned}
$$

ahora, empleando la ecuación (40), resulta

$$
\begin{aligned}
F_{23 y} & =m_{A} a_{A y}-F_{43 y} \\
& =m_{A} a_{A y}-\left(m_{3 b} a_{B x}+m_{4} a_{B x}\right) \tan \phi
\end{aligned}
$$

La componente $F_{43 y}$ se simplifica recordando las ecuaciones (36) y (40), resultando,

$$
F_{43 y}=\left(m_{3 b} a_{B x}+m_{4} a_{B x}\right) \tan \phi
$$

Es interesante observar cómo las componentes en la ordenada $y$, dependen de las aceleraciones en la abscisa $x$ y viceversa en las dos últimas expresiones, son estas componentes las que transfieren el movimiento horizontal en vertical y generan las fuerzas de sacudimiento. En este contexto esta interpretación coincide con la definición de Norton (2009).

Hasta ahora las tres últimas ecuaciones están expresadas en términos de las masa parciales puntuales, el siguiente paso es expresar éstas ecuaciones en términos de las masas puntuales totales. Para esto se recuerdan las expresiones de la ecuación (32).

De esta forma, las ecuaciones (48), (50) y (51) se escriben ahora por,

$F_{23 x}=m_{A} a_{A x}+m_{B} a_{B x}$

$F_{23 y}=m_{A} a_{A y}-m_{B} a_{B x} \tan \phi$

$F_{43 y}=m_{B} a_{B x} \tan \phi$

Estas formas más compactas de las fuerzas que producen el sacudimiento, dependen de dos aceleraciones, $a_{A}$ y $a_{B}$.

De las ecuaciones (46) y (47) que corresponden con las fuerzas inerciales, se establecen entonces las fuerzas de sacudimiento,

$$
\begin{aligned}
F_{s x} & =-F_{i x}=F_{23 x}=m_{A} a_{A x}+m_{B} a_{B x} \\
F_{s x} & =-F_{i x}=F_{23 x}=-F_{32 x} \\
F_{s y} & =-F_{i y}=F_{23 y}+F_{43 y}= \\
& =m_{A} a_{A y}-m_{B} a_{B x} \tan \phi+m_{B} a_{B x} \tan \phi \\
& =m_{A} a_{A y}
\end{aligned}
$$

Es interesante observar cómo las fuerzas de sacudimiento $F_{s x}$ y $F_{s y}$, tienen formas simples de interpretar, $F_{s x}$ es el resultado de las masas puntuales en movimiento, $m_{A}$ y $m_{B}$, multiplicada cada una por su correspondiente aceleración. Este resultado es el que prevé la Segunda Ley de Newton, pero se ha llegado hasta él, a partir de evaluar las fuerzas internas y las correspondientes fuerzas de sacudimiento.

Para $F_{s y}$, se tiene la misma situación, la única masa que se desplaza en el plano del movimiento por arriba de la horizontal es $m_{A}$. 
Con la información obtenida hasta ahora es posible expresar de forma completa las variaciones de las fuerzas de sacudimiento en el tiempo, esto se logra al sustituir las expresiones de la aceleración en cada caso.

Las ecuaciones (50) y (51) están en términos de las aceleraciones $a_{A}$ y $a_{B}$. A continuación se presentas las expresiones de éstas aceleraciones.

$\boldsymbol{a}_{A}=\frac{d^{2} r}{d t^{2}}=-r \omega^{2} \cos \omega t \hat{\boldsymbol{\imath}}-r \omega^{2} \operatorname{sen} \omega t \hat{\boldsymbol{\jmath}}$

de donde se determinan las componentes respectivas,

$a_{A x}=-r \omega^{2} \cos \omega t, a_{A y}=-r \omega^{2} \operatorname{sen} \omega t$

La ecuación (15) que se obtuvo al inicio de estos desarrollos corresponde con la posición del $x$ pistón. Al obtener su segunda derivada, se obtendrá la expresión de la aceleración, esto es,

$\ddot{x} \cong-r \omega^{2}\left(\cos \omega t+\frac{r}{l} \cos 2 \omega t\right)$

Esta aceleración corresponde con la aceleración del pistón, por tanto,

$a_{B}=a_{B x} \cong-r \omega^{2}\left(\cos \omega t+\frac{r}{l} \cos 2 \omega t\right)$

\section{Par de sacudimiento}

El par de sacudimiento se determinó con la ecuación (34), esta cantidad está en función de las fuerzas $F_{32 x}$ y $F_{32 y}$, y por el ángulo $\theta$. Su valoración es importante porque contiene información de un efecto de rotación sobre el plano del movimiento y es sobre esta cantidad sobre la cual se analiza el balanceo del mecanismo.

Sustituyendo en la ecuación (34) las ecuaciones (52) y (53), se obtiene

$T_{12}=-m_{A} a_{A x} r \operatorname{sen} \theta-m_{B} a_{B x} r \operatorname{sen} \theta$
$+m_{A} a_{A y} r \cos \theta+m_{B} a_{B x} \tan \phi r \cos \theta$

Sustituyendo las expresiones de la ecuación (58) y recordando que $\theta=\omega t$, resulta

$$
\begin{gathered}
T_{12}=m_{A}\left(r \omega^{2} \cos \omega t\right) r \operatorname{sen} \omega t \\
+m_{B} a_{B x} r \operatorname{sen} \omega t \\
-m_{A}\left(r \omega^{2} \operatorname{sen} \omega t\right) r \cos \omega t \\
+m_{B} a_{B x} \tan \phi r \cos \omega t
\end{gathered}
$$

Lo anterior se reduce a,

$$
T_{12}=m_{B} r a_{B x}(\operatorname{sen} \omega t+\tan \phi \cos \omega t)
$$

Sustituyendo la ecuación (8) en la ecuación (63), resulta,

$$
T_{12}=m_{B} r a_{B x} \operatorname{sen} \omega t\left(1+\frac{\frac{r}{l} \cos \omega t}{\sqrt{1-\left(\frac{r}{l} \operatorname{sen} \omega t\right)^{2}}}\right)(64)
$$

La aproximación en series del término del inverso del radical es,

$\frac{1}{\sqrt{1-\left(\frac{r}{l} \operatorname{sen} \omega t\right)^{2}}} \cong 1+\frac{r^{2}}{2 l r^{2}}(\operatorname{sen} \omega t)^{2}$

Al sustituir la expresión (63) en (62), resulta,

$T_{12} \cong m_{B} r a_{B x} \operatorname{sen} \omega t\left(1+\frac{r}{l} \cos \omega t+\right.$ $\left.\frac{r^{2}}{2 l^{2}} \frac{r}{l} \cos \omega t(\operatorname{sen} \omega t)^{2}\right)$

La relación $r / l$, puede alcanzar valores muy pequeños, por lo que los términos asociados con estos productos, para potencias mayores a dos, se pueden retirar de la expresión del par de sacudimiento, el resultado es,

$T_{12} \cong m_{B} r a_{B x} \operatorname{sen} \omega t\left(1+\frac{r}{l} \cos \omega t\right)$

Ahora se considera la expresión de la aceleración $a_{B x}$ de la ecuación (60) y se sustituye en la ecuación (66),

$T_{12} \cong-r^{2} \omega^{2} m_{B}\left(\cos \omega t+\frac{r}{l} \cos 2 \omega t\right)(1+$

$\left.\frac{r}{l} \cos \omega t\right) \operatorname{sen} \omega t$

El desarrollo de los productos, produce nuevamente potencias del orden del cuadrado del cociente de $r / l$, el resultado es

$$
\begin{aligned}
& T_{12} \cong-r^{2} \omega^{2} m_{B}\left(\cos \omega t+\frac{r}{l} \cos ^{2} \omega t+\right. \\
& \left.\frac{r}{l} \cos 2 \omega t\right) \operatorname{sen} \omega t
\end{aligned}
$$

La identidad trigonométrica siguiente,

$$
\cos ^{2} \omega t=\frac{1}{2} \cos 2 \omega t+\frac{1}{2}
$$

Se sustituye en la ecuación (69) y el resultado es,

VÁZQUEZ-GONZÁLEZ, Benjamín, JIMÉNEZ-RABIELA, Homero, RAMÍREZ-CRUZ, José Luis y GARCÍA-SEGURA, Pedro. Desarrollo didáctico complementario de las expresiones analíticas de las acciones de sacudimiento en las máquinas de combustión interna. Revista de Tecnología y Educación. 2019 
$T_{12} \cong-r^{2} \omega^{2} m_{B}\left(\cos \omega t+\frac{r}{l}\left(\frac{1}{2} \cos 2 \omega t+\right.\right.$

$\left.\left.\frac{1}{2}\right)+\frac{r}{l} \cos 2 \omega t\right) \operatorname{sen} \omega t$

Desarrollando lo anterior,

$$
\begin{aligned}
& T_{12} \cong-r^{2} \omega^{2} m_{B}\left(\cos \omega t+\frac{r}{2 l} \cos 2 \omega t+\frac{r}{2 l}+\right. \\
& \left.\frac{r}{l} \cos 2 \omega t\right) \operatorname{sen} \omega t
\end{aligned}
$$

Finalmente, simplificando resulta,

$$
\begin{aligned}
& T_{12} \cong-r^{2} \omega^{2} m_{B}\left(\cos \omega t+\frac{r}{2 l}+\right. \\
& \left.\frac{3 r}{2 l} \cos 2 \omega t\right) \operatorname{sen} \omega
\end{aligned}
$$

Este es el resultado reportado por Norton (2009), también por Uicker et. al. (2003), desde la perspectiva de las fuerzas de inercia, el procedimiento seguido en este trabajo ha seguido los fundamentos de la dinámica, y también de las estrategias sobre el efecto de los términos de las expresiones en los desarrollos de series de potencias.

\section{Conclusiones}

En este trabajo se presentan los resultados del análisis dinámico de las fuerzas que se presentan en un mecanismo biela-manivelacorredera. Se presentan desarrollos algebraicos basados en esquemas realizados con detalle, a fin de asegurar de manera concreta y completo el análisis realizado. Una aportación presentada en este trabajo consiste en el desarrollo alterno, de las aproximaciones en series de potencias de los términos trascendentales que modelan de manera concreta a las expresiones con las que se evalúan las fuerzas y los pares de sacudimiento.

El desarrollo de dichas aproximaciones se maneja en conjunto con las representaciones de los modelos de masa puntuales equivalente, de los eslabones del mecanismo. La estrategia didáctica seguida en este trabajo, consiste desarrollar las expresiones obtenidas de los modelos dinámicos, acompañadas de la interpretación que permite unir conceptualmente los desarrollos analíticos, para obtener un modelo completo.

\section{Agradecimiento}

Los autores agradecemos el apoyo institucional, de la Universidad Autónoma Metropolitana y de la Unidad Azcapotzalco, en particular de la División de Ciencias Básicas e Ingeniería y del Departamento de Energía.

\section{Referencias}

$\mathrm{ABB}$ (2016), http://www.abb.com/cawp/seitp202/714dc9a01 89e50bdc1258026004f84bf.aspx

Cleghorn, W. L., (2005). Mechanics of Machines, USA, Oxford University Press.

Erdman, A. G., Sandor, G. N., Kota, Sridhar. (2001). Mechanism Design. Analysis and Synthesis, Vol. 1.USA. Prentice Hall.

Menchaca Torre, Hilda Lizette y Mendoza Domínguez Alberto. (2013). Desempeño de un vehículo híbrido y su contraparte de combustión interna bajo condiciones de manejo de una ciudad mexicana. Rev. Int. Contam. Ambie. 29 (2) 219-230.

Norton, R. (2009). Kinematics and Dynamics of Machinery, USA: McGraw-Hill Education.

Uicker J. J. Jr., Gordon R. P. y Shigley J. E. (2003). Theory of Machines and Mechanisms, USA. Oxford University Press.

Wilson C. E. y Sadler J. P. (2002). Kinematics and Dynamics of Machinery, USA. Pearson. 